\title{
Modeling of Stardust Entry at High Altitude, Part 2: Radiation Analysis
}

\author{
Iain D. Boyd* \\ University of Michigan, Ann Arbor, Michigan 48109-2140 \\ and \\ Peter Jenniskens $\ddagger$ \\ SETI Institute, Mountain View, California 94043 \\ DOI: $10.2514 / 1.37357$
}

\begin{abstract}
During entry of the Stardust sample return capsule, measurements of the radiation emitted from the shock-heated flow were obtained with a number of instruments. These instruments viewed the sample return capsule from an airplane located several hundred kilometers from the vehicle. The present study analyzes the radiation generated at two different high-altitude conditions. The flowfields are simulated using both continuum (computational fluid dynamics) and particle (direct simulation Monte Carlo) methods. The flow solutions provide input to a nonequilibrium radiation model to compute line-of-sight spectra that are compared with high-resolution data taken with the Echelle spectrograph during the Stardust entry. Comparisons between simulation and measurements are presented for air spectral features and for metal atomic lines believed to originate from evaporation of the vehicle surface. The comparisons make it possible to identify specific aspects of the air chemistry modeling that require further work. In addition, analysis of the metal spectra provides insight into the likely sources of these impurities.
\end{abstract}

\section{Introduction}

A UNIQUE aspect of the Earth entry portion of the Stardust mission involved the measurement, by a number of different instruments, of radiation emitted from the shock-heated air. The study reported here analyzes the flowfields and resulting emissions for two different high-altitude points on the Stardust entry trajectory. The forebody thermal protection material used on Stardust was a phenolic-impregnated carbon ablator (PICA) that has also been selected as a candidate material for use on NASA's Orion crew exploration vehicle. The combination of having an entry velocity similar to Mars return and the use of PICA as heat shield material makes it of significant interest to perform detailed analyses of the Stardust sample return capsule (SRC) entry even after the successful completion of its mission.

A number of studies of the aerothermodynamics of the Stardust SRC were performed before the mission [1-4] . These studies conducted flowfield analyses using both continuum computational fluid dynamics (CFD) methods (by solving the Navier-Stokes equations) and particle methods (using the direct simulation Monte Carlo method DSMC [5]). However, the DSMC study [3] was focused on the high-altitude aerodynamics of the capsule and did not include the effects of ionization reactions. Recent studies have compared CFD and DSMC simulation results for Stardust at high altitudes where ionization was included [6,7].

The primary goal of this study is to perform high-altitude analyses of the radiation environment around the Stardust SRC. Flowfield computations are performed with both continuum and particle methods. Direct comparisons between the solutions provided by these techniques allow the evaluation of differences between these approaches for modeling the strongly nonequilibrium

Presented as Paper 2008-1218 at the AIAA Aerospace Sciences Meeting, Reno, NV, 6-9 January 2008; received 29 February 2008; revision received 6 February 2009; accepted for publication 26 April 2009. Copyright $\odot 2009$ by the American Institute of Aeronautics and Astronautics, Inc. All rights reserved. Copies of this paper may be made for personal or internal use, on condition that the copier pay the $\$ 10.00$ per-copy fee to the Copyright Clearance Center, Inc., 222 Rosewood Drive, Danvers, MA 01923; include the code 0022-4650/10 and $\$ 10.00$ in correspondence with the CCC.

${ }^{*}$ Professor, Department of Aerospace Engineering, 1320 Beal Avenue. Associate Fellow AIAA

${ }^{\dagger}$ Research Scientist, Carl Sagan Center, 515 North Whisman Road. Senior Member AIAA. thermochemical phenomena generated under highly energetic entry conditions. Such comparisons for the flowfields used to compute the radiation in the present study are described in detail in a companion paper []].

The geometry of the Stardust SRC, the entry conditions chosen for study, and the spectral data are first described. The two numerical methods employed in the study for flowfield computation are then outlined. The radiation associated with the flowfields is computed using the NEQAIR code [9], which is also described briefly. The radiation results are then presented and discussed in detail. Final conclusions are formulated.

\section{Flow Conditions and Spectral Measurements}

The geometry of the Stardust SRC forebody consists of a $60 \mathrm{deg}$ half-angle, spherically blunted cone with a nose radius of $0.2202 \mathrm{~m}$. A small portion of the shoulder is included in the computations that has a radius of $0.02 \mathrm{~m}$. The flow and surface conditions for the two altitudes considered are listed in Table 1 . The Knudsen numbers are in the near-continuum regime where only small differences would be expected between CFD and DSMC simulation results.

This study compares computed spectra with data from the Echelle instrument [10], a miniature Echelle spectrograph that provided a high spectral resolution over a wide wavelength range from 360 to $880 \mathrm{~nm}$, increasing from full width at half-maximum $\mathrm{FWHM}=0.3$ to $0.9 \mathrm{~nm}$ over this range, and that obtained 100 spectra over the altitude range of $82-69 \mathrm{~km}$. The spectra contain a number of interesting features, including 1) an underlying continuum signal from the heated surface of the Stardust SRC, 2) atomic lines of airplasma species, 3) atomic lines of metals assumed to originate from the white paint applied to the exterior of the thermal protection system (TPS), and 4) band structures from air molecules and cyanide. Details of the processing of the spectra and discussions of the measured data are provided by Jenniskens [10].

\section{Flowfield Simulations}

The simulation methods and flowfield results are described in detail by Boyd et al. [8]. Here, a brief overview is provided. The two different numerical approaches applied are as follows: 1) the CFD solution of the continuum Navier-Stokes equations using the DPLR code, and 2) particle-based DSMC computation. In each case, an 11species, 19-reaction, thermochemical nonequilibrium approach is 
Table 1 Freestream and surface parameters

\begin{tabular}{lcc}
\hline \hline & \multicolumn{2}{c}{ Altitude, $\mathrm{km}$} \\
Parameter & 81 & 71 \\
\hline Freestream temperature, $\mathrm{K}$ & 217.6 & 221.6 \\
Freestream number density, & $2.643 \times 10^{20}$ & $1.156 \times 10^{21}$ \\
$\quad$ molecules $/ \mathrm{m}^{3}$ & & \\
Freestream velocity, m/s & 12,385 & 12,063 \\
Freestream $\mathrm{O}_{2}$ mole fraction, \% & 23.67 & 23.67 \\
Freestream $\mathrm{N}_{2}$ mole fraction, $\%$ & 76.23 & 76.23 \\
Freestream Knudsen number, & 0.005 & 0.001 \\
Surface temperature, K & 2000 & 2700 \\
\hline \hline
\end{tabular}

adopted. The wall temperature is assumed fixed in each case at the values listed in Table 1 . The PICA wall material is assumed to be fully catalytic to ions (which recombine into their neutral atoms and molecules) and atoms (which recombine into molecules). Ablation processes are not included in these studies.

The continuum computations are performed using the DPLR CFD code $[11,12]$. DPLR is a parallel multiblock finite volume code that solves the Navier-Stokes equations including finite rate chemistry and the effects of thermal nonequilibrium. Three separate temperatures are modeled for the translational, rotational, and vibrational modes. Rotational relaxation is modeled using the relaxation time based on the Parker model [13]. Vibrational relaxation is modeled using a Landau-Teller formulation, where relaxation times are obtained from Millikan and White [14], assuming simple harmonic oscillators, with the high-temperature correction of Park [15]. The 19-reaction, finite rate air chemistry model uses a standard set of rates $[16,17]$. The backward rates are computed using the forward rates and equilibrium constants evaluated using the van't Hoff equation.

The baseline DPLR case (DPLR-b) employs the standard Park [15] two-temperature model for dissociation, in which the controlling temperature $T=T_{t}^{0.5} \times T_{v}^{0.5}$ and all other reactions are controlled solely by the translational temperature. Because it is not possible to use the standard direct ionization rates in DSMC, a second modified DPLR solution (DPLR-m) is obtained using the rates that are employed in the DSMC computations. Further, to address differences in chemistry modeling between DPLR and DSMC, the second DPLR computation makes use of different controlling temperatures $T=T_{t}^{n} \times T_{v}^{1-n}$ for both dissociation and ionization reactions. For dissociation, the modified DPLR simulation uses $n=0.3$. For direct ionization, DPLR uses $n=0.0$ assuming that the vibrational temperature in DPLR is a better approximation to the electron temperature than the translational temperature. In the presentation of results, the baseline DPLR case is indicated by "b" and the modified chemistry case by " $m$."

The particle computations employ a DSMC code developed specifically for hypersonic, ionized flow simulations [18]. Models are implemented for rotational [19] and vibrational [20] energy exchange that are consistent with those employed in DPLR. Most of the chemical reactions are simulated using the total collision energy (TCE) model except for the dissociation of nitrogen and oxygen. These reactions employ the vibrationally favored dissociation model [21].

Most of the forward rate coefficients employed in the DSMC computations are the same as those employed by DPLR. The exceptions are the two direct ionization reactions

$$
\begin{aligned}
& \mathrm{N}+\mathrm{E}^{-} \rightarrow \mathrm{N}^{+}+2 \mathrm{E}^{-} \\
& \mathrm{O}+\mathrm{E}^{-} \rightarrow \mathrm{O}^{+}+2 \mathrm{E}^{-}
\end{aligned}
$$

It is not possible to use in the DSMC TCE chemistry model the low, negative temperature exponents of the rates for these two reactions usually employed by DPLR. The rates employed in the DSMC and the modified DPLR simulations are those reported by Wilson [22]. The backward steps of all chemical reactions are modeled using the approach described by Boyd [23] that provide consistency with the CFD approach.
Detailed discussions of the flowfield results may be found in the companion article [8]. Here, a brief summary is provided to help to understand the computed spectra. In terms of radiation, the flow properties of most interest are the various temperatures and the number densities of atomic nitrogen and oxygen, molecular nitrogen ions, and electrons. The atoms and nitrogen ions produce significant spectral features in the wavelength range interrogated by the Echelle instrument. The electrons participate directly in the modeling of the excitation processes.

At $81 \mathrm{~km}$, in comparison with the baseline DPLR solution, the shock wave predicted by DSMC is significantly thicker and the peak translational temperature from DSMC is significantly higher. The modified DPLR case is in much better agreement with the DSMC results, with significant increases in the peak temperatures for each of the translational, rotational, and vibrational modes. In addition, the shock thickness is increased substantially. Note that DSMC provides the translational temperature of the electrons, whereas DPLR assumes that the electrons have the same translational temperature as the bulk gas. The atom number densities predicted by the three simulations are in generally good agreement, except at distances close to the capsule surface where temperature jump predicted by DSMC leads to lower values. As illustrated along the stagnation streamline in Fig. 1, the baseline DPLR case predicts a very low level of $\mathrm{N}_{2}^{+}$in comparison to the other two simulations. On the other hand, as shown in Fig. 2, the baseline DPLR solution predicts a peak electron number density that is about a factor of 25-30 higher than the peak predictions from the other two simulations.

At $71 \mathrm{~km}$, the two DPLR solutions are much less sensitive to chemistry modeling at this higher density flow condition, as

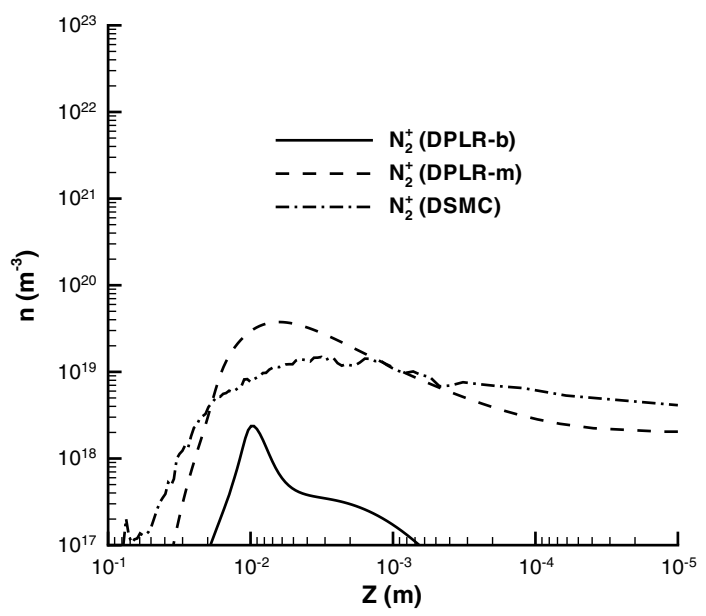

Fig. 1 Profiles of molecular nitrogen ion number density along the stagnation streamline for the Stardust return capsule at $81 \mathrm{~km}$.

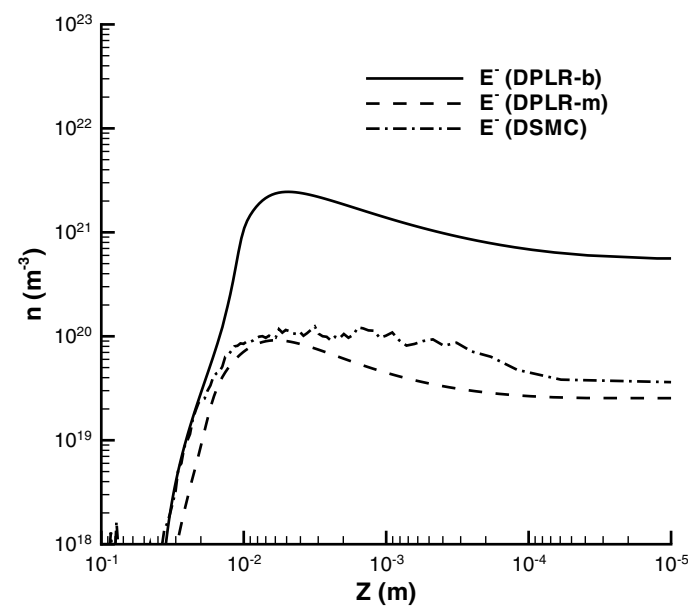

Fig. 2 Profiles of electron number density along the stagnation streamline for the Stardust return capsule at $81 \mathrm{~km}$. 


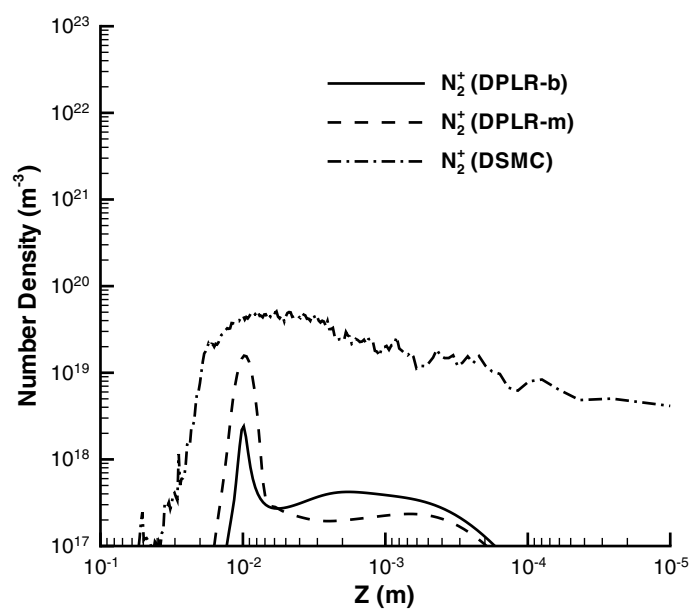

Fig. 3 Profiles of molecular nitrogen ion number density along the stagnation streamline for the Stardust return capsule at $71 \mathrm{~km}$.

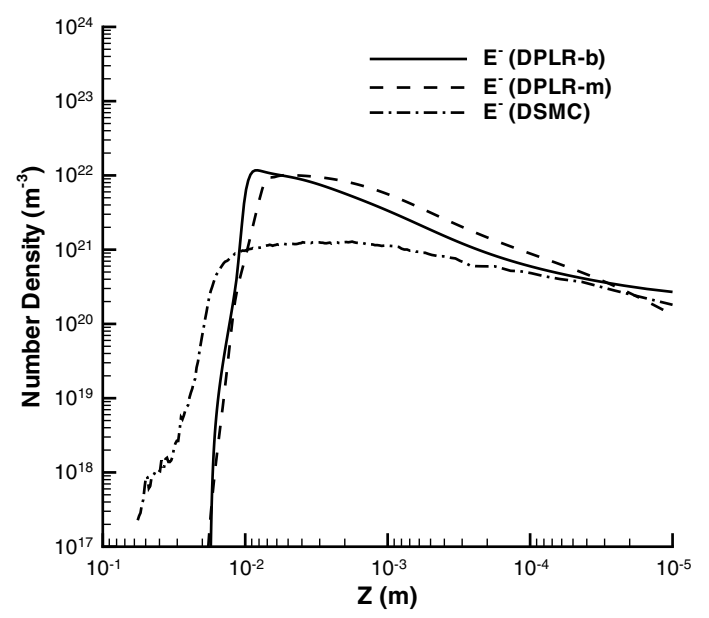

Fig. 4 Profiles of electron number density along the stagnation streamline for the Stardust return capsule at $71 \mathrm{~km}$.

expected. There are still some significant differences between the DSMC and DPLR results, especially for the number densities of $\mathrm{N}_{2}^{+}$ and $\mathrm{E}^{-}$, as shown in Figs. $\underline{3}$ and 4 . Specifically, DSMC predicts a much higher peak density for $\mathrm{N}_{2}^{+}$and a much lower level of $\mathrm{E}^{-}$.

\section{Radiation Analysis}

Line-of-sight radiation is computed from the flowfield solutions using the nonequilibrium radiation code NEQAIR [9]. To populate the excited states, NEQAIR provides options to employ either an equilibrium assumption (Boltzmann) or a finite rate approach under the assumption of a quasi-steady state (QSS). In the present work, the QSS approach is employed for air species. Because of a lack of published excitation rates required by the QSS model, the Boltzmann model is employed for metal atomic lines. NEQAIR takes as input the temperatures of the translational, rotational, and vibrational modes, the electron temperature, and the number densities of all chemical species. Although the DSMC computation simulates these four different temperatures, DPLR does not simulate the electron temperature. In computing spectra based on DPLR solutions, the sensitivity to using either the translational or vibrational temperature to represent the electron temperature is investigated. In all cases, each flowfield line of sight begins at the vehicle surface and extends to the freestream, as this is the direction of propagation of the emission detected by the Echelle instrument. All spectra are presented in units of flux density which requires the computed spectra to be corrected for the solid angle subtended by the Stardust capsule at the instrument.

\section{Spectral Results}

The presentation of radiation analyses are divided into separate sections focused on air species and metal species.

\section{A. Emissions from Air Species}

The full spectrum measured by the Echelle instrument at $81 \mathrm{~km}$ is shown in Fig. 5. Recall that the measured spectra include a continuum from the heated capsule surface, and this is not included in the computed spectra. This approach is taken due to the difficulty of fitting the high-altitude measured spectra to a single temperature. The Echelle data are compared in Fig. 5 with three simulated spectra evaluated along the line of sight corresponding to the stagnation streamline of the flow: 1) DSMC, 2) baseline DPLR with the electron temperature $T_{e}$ set to the translational temperature $T_{t}$, and 3 ) baseline DPLR with the electron temperature set to the vibrational temperature $T_{v}$. The comparison indicates significant differences between the three computed spectra. As expected, because the translational temperature is always higher than the vibrational temperature, the DPLR-based spectra using $T_{e}=T_{t}$ have significantly higher levels of emission.

In Fig. 6 the same comparison is made, only here using the two spectra obtained from the modified DPLR simulation. Again, there is

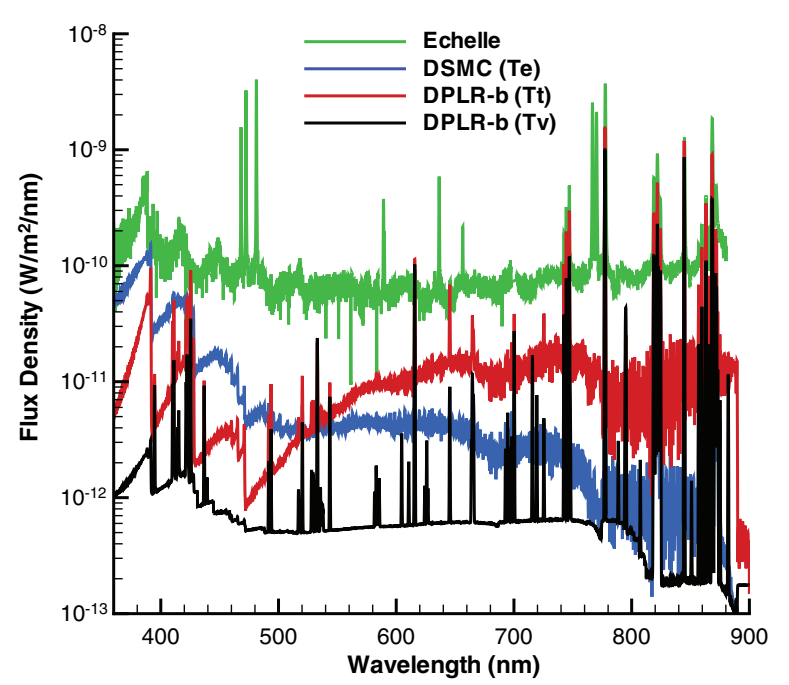

Fig. 5 Comparisons of measured and computed spectra across the full range of the Echelle instrument at $81 \mathrm{~km}$ : DPL R-b = baseline chemistry.

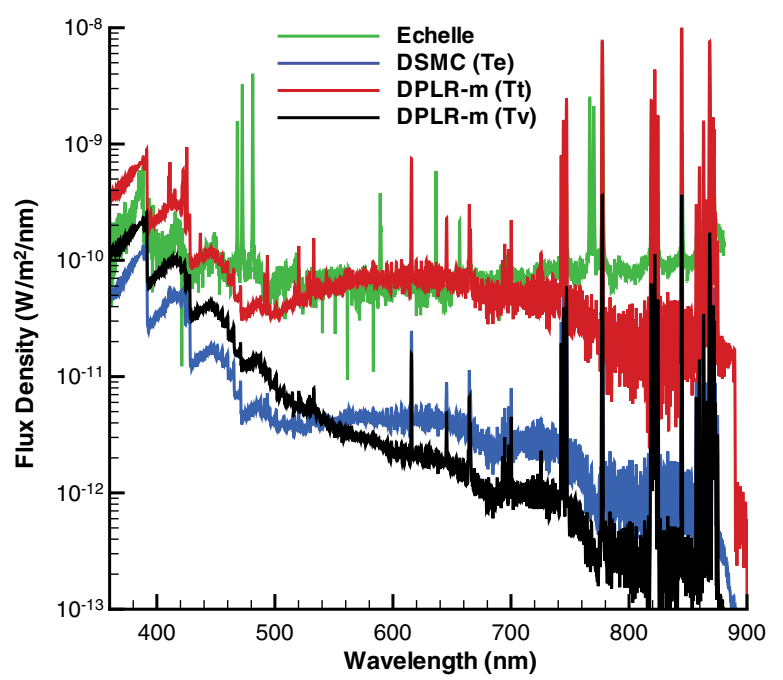

Fig. 6 Comparisons of measured and computed spectra across the full range of the Echelle instrument at $81 \mathrm{~km}$ : DPLR-m $=$ modified chemistry. 
significant variation between the three simulation results and, by comparison with the DPLR spectra in Fig. 5, it is clear that there is also significant variation among the four DP LR results.

To investigate the spatial variation of the radiation, flowfield profiles from all simulations are extracted at several radial locations. The line-of-sight spectra based on some of these profiles are compared. In Fig. 7, a comparison is made for four different radial profiles for the baseline DPLR simulation using $T_{e}=T_{t}$. There is relatively little variation among these profiles with a maximum difference of less than $20 \%$. The same comparison is made in Fig. 8 with emission evaluated using $T_{e}=T_{v}$. The change in spectra with radius is much more significant in this case, with more than a factor of 2 variation. Similar levels of radial variation are found for the spectra obtained from the modified DPLR flowfields. Finally, the radial dependence of the DSMC-based spectra is shown in Fig. 9. Here, the maximum difference between the spectra is as high as a factor of 4 .

In general, the DPLR-based spectra computed using $T_{e}=T_{t}$ show a small radial variation, whereas the DPLR spectra using $T_{e}=T_{v}$ and the DSMC spectra show considerable variation. The difference between the trends in the DPLR results is explained by the fact that the vibrational temperature generally increases with radius because the flow has more time to undergo vibrational relaxation. At the same time, the region of high vibrational temperature becomes thicker

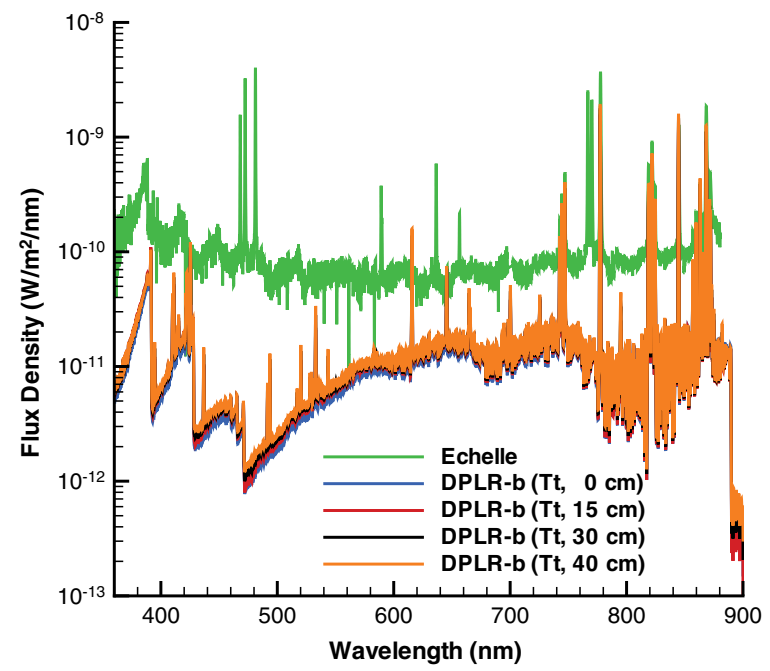

Fig. 7 Radial variation of computed spectra across the full range of the Echelle instrument at $81 \mathrm{~km}$ : DPL R-b = baseline chemistry.

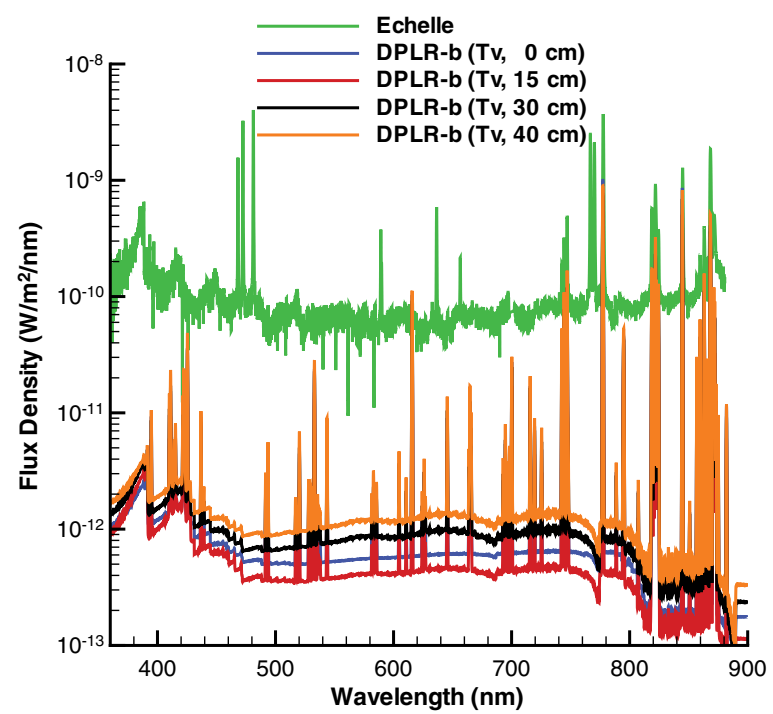

Fig. 8 Radial variation of computed spectra across the full range of the Echelle instrument at $81 \mathrm{~km}$ : DPL R-b = baseline chemistry.

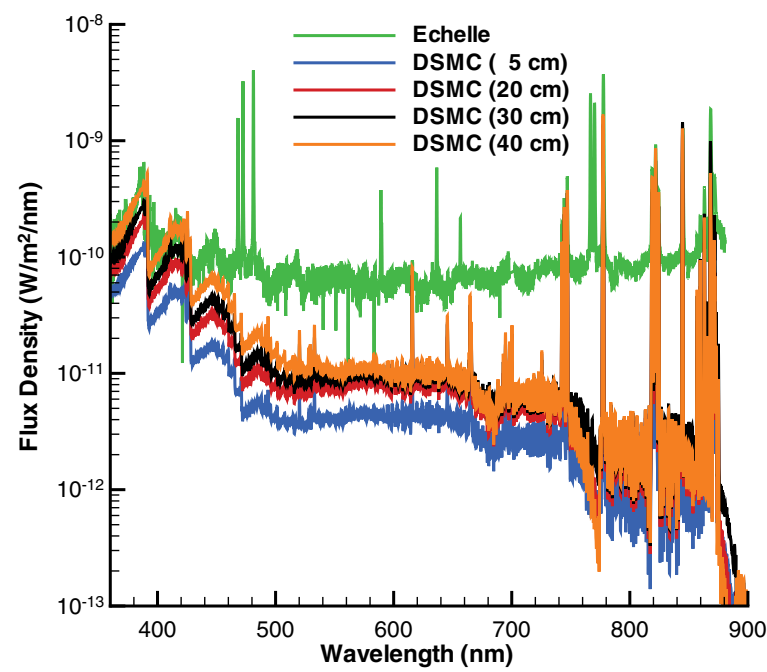

Fig. 9 Radial variation of computed spectra across the full range of the Echelle instrument at $81 \mathrm{~km}$.

away from the stagnation streamline. Similarly, in the DSMC solution, the peak electron temperature and shock-layer thickness both increase with radius due to analogous effects. These trends can be seen in Fig. 10, which shows contours of vibrational temperature from the modified DPLR case (upper) and electron temperature contours from DSMC (lower). By comparison, the translational temperature contours from the DPLR simulations show relatively little variation in magnitude and thickness with radius [8].

Viewing the Stardust entry from a distance of several hundred kilometers, the Echelle instrument of course has no spatial resolution. Therefore, area weighting is employed to derive spatially averaged, computed spectra that are compared with the measured data in the remainder of this study. In all cases, 10 lines of sight are extracted from the flowfield parallel to the flow axis. The associated spectrum is computed for each line of sight, which are then area averaged to determine the final computed spectra.

To better assess the various spectral predictions, comparisons are next provided over narrower wavelength ranges. In Fig. 11, spectra are shown in the range of $370-420 \mathrm{~nm}$. In the measured spectra, this region contains a strong signal attributed to cyanide $(\mathrm{CN})$ in the range of $385-389 \mathrm{~nm}$, as well as a peak at around $391.4 \mathrm{~nm}$ attributed to a bandhead of the $\mathrm{N}_{2}^{+}$molecular system. None of the flowfield simulations (DPLR and DSMC) include carbon chemistry, so that comparison with the $\mathrm{CN}$ emission is not possible. In addition, the measured $\mathrm{CN}$ emission appears to overwhelm some of the $\mathrm{N}_{2}^{+}$ emission features. Note that there are also a pair of lines in the Echelle

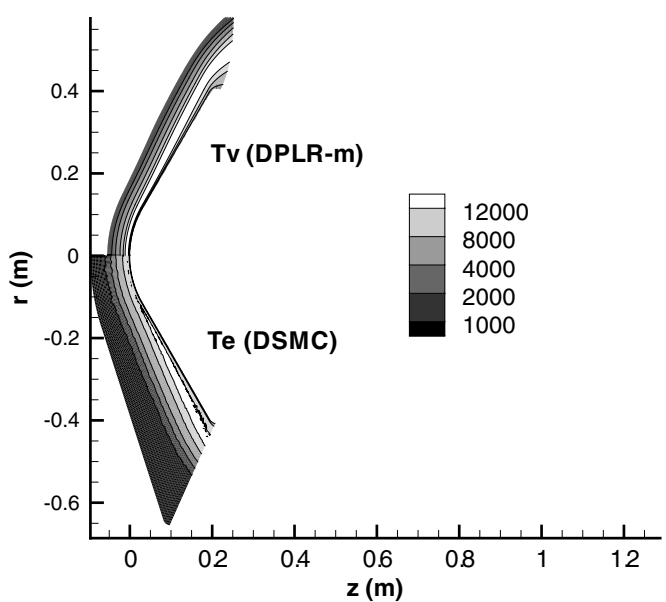

Fig. 10 Contours of vibrational temperature (in Kelvin) obtained using the modified DPLR case (upper) and contours of electron temperature from DSMC (lower) at $81 \mathrm{~km}$. 


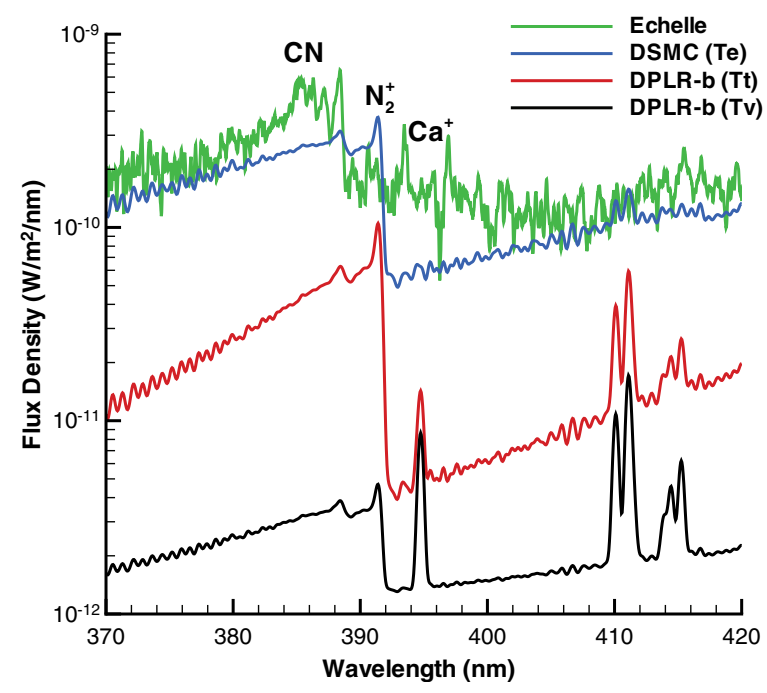

Fig. 11 Comparisons of measured and computed spectra focusing on air molecular bands at $81 \mathrm{~km}$ : DPL R-b = baseline chemistry.

data attributed to calcium ions that are not included in any of the analyses. The DPLR spectra shown in Fig. 11 are for the baseline chemistry. In comparison to the Echelle data, the DSMC spectrum slightly overpredicts the $\mathrm{N}_{2}^{+}$bandhead, whereas the DPLR spectrum using $T_{e}=T_{t}$ slightly underpredicts it. The DPLR spectrum using $T_{e}=T_{v}$ significantly underpredicts the feature. The DPLR spectra shown in Fig. 12 are from the modified chemistry simulation. In this case, the DPLR result using $T_{e}=T_{v}$ is in good agreement with the DSMC result and the Echelle data. These comparisons indicate that there is significant uncertainty in the prediction of $\mathrm{N}_{2}^{+}$chemistry at this flow condition, with the DSMC and modified DPLR cases providing best agreement with the data.

In Fig. 13, spectra are shown in the range of $810-880 \mathrm{~nm}$ that contain several lines of atomic nitrogen and oxygen. Careful examination of these atomic spectral features indicates that the baseline DPLR result using $T_{e}=T_{t}$ is in good agreement with the DSMC spectrum and that both agree well with the measured Echelle data. By comparison, the baseline DPLR result using $T_{e}=T_{v}$ underpredicts the atomic line intensities. The corresponding comparison is provided in Fig. 14 for the modified DPLR simulation. In this case, the DPLR result using $T_{e}=T_{t}$ significantly overpredicts the atomic line intensities, whereas the DPLR solution with $T_{e}=T_{v}$ provides good agreement with both DSMC and the Echelle data.

Similar comparisons are made in the wavelength range of 740 $780 \mathrm{~nm}$ in Figs. 15 and 16. Strong lines of potassium are observed in

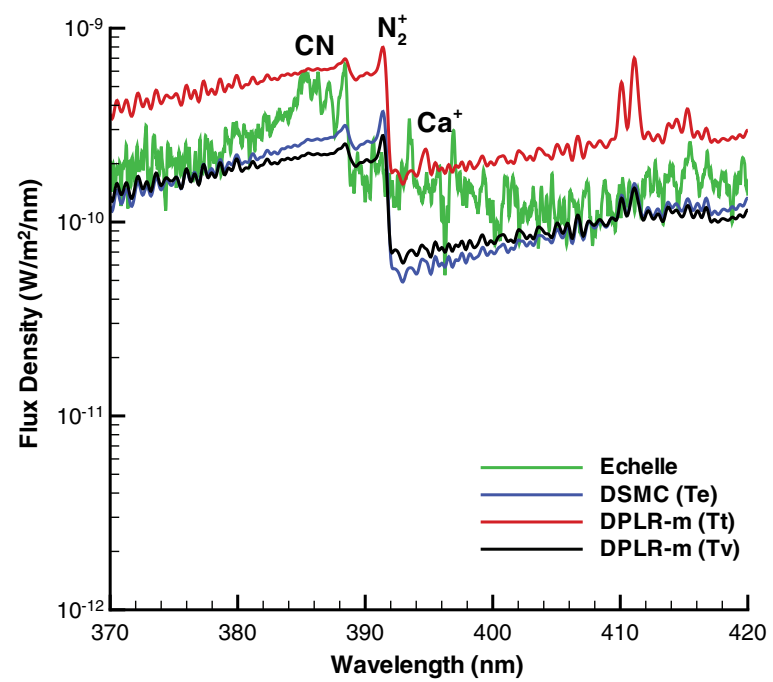

Fig. 12 Comparisons of measured and computed spectra focusing on air molecular bands at $81 \mathrm{~km}$ : DPL R-m = modified chemistry.

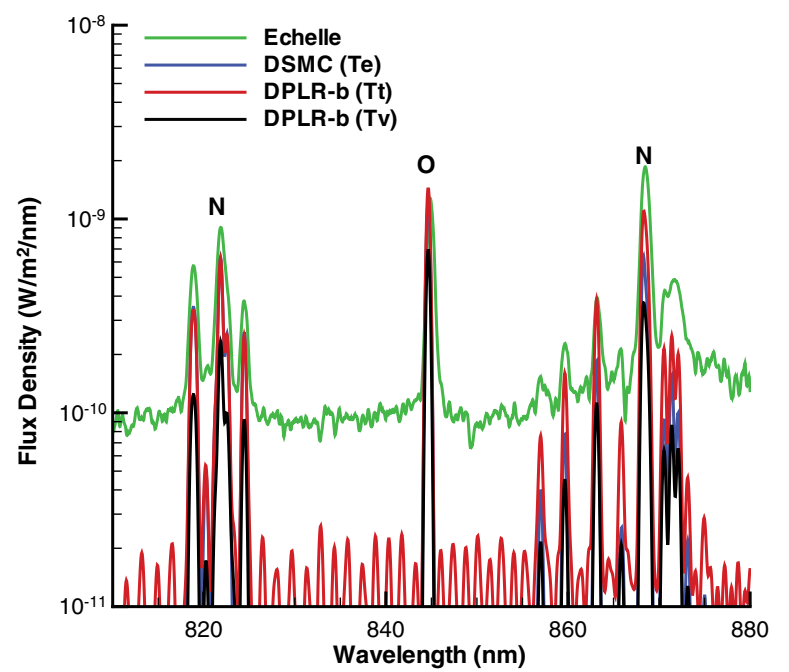

Fig. 13 Comparisons of measured and computed spectra focusing on air atomic lines at $81 \mathrm{~km}$ : DPL R-b = baseline chemistry.

the Echelle data in this portion of the spectrum. Focusing here on the air atomic lines, the conclusions are again that DSMC agrees quite well with the baseline DPLR solution using $T_{e}=T_{t}$, the modified DPLR solution using $T_{e}=T_{v}$, and with the Echelle data.

The results obtained at $71 \mathrm{~km}$ are now considered. The freestream condition at this altitude is 5 times denser than that at $81 \mathrm{~km}$. In Fig. 17, the full spectrum measured by the Echelle instrument at $71 \mathrm{~km}$ is compared with three simulation results along the stagnation streamline of the flow: 1) DSMC, 2) modified DPLR with the electron temperature $T_{e}$ set to the translational temperature $T_{t}$, and 3) modified DPLR with the electron temperature set to the vibrational temperature $T_{v}$. The comparison indicates a significant reduction in the sensitivity of the DPLR-based spectra to the electron temperature. In addition, the DPLR-based spectra using the baseline DPLR chemistry model give almost identical profiles to those shown here. The reduced sensitivity to chemistry modeling and excitation temperature associated with the DPLR results is qualitatively anticipated at the denser, lower altitude condition. Thus, for the remainder of the $71 \mathrm{~km}$ analysis, only DPLR-based spectra using the modified chemistry modeling are shown. Note, however, that there are clearly still significant differences between the DSMC and DPLR-based spectra in Fig. 17.

Radial variation of the spectra computed at $71 \mathrm{~km}$ is very similar to that discussed earlier at $81 \mathrm{~km}$. The spectra presented next are again spatially averaged across the face of the capsule.

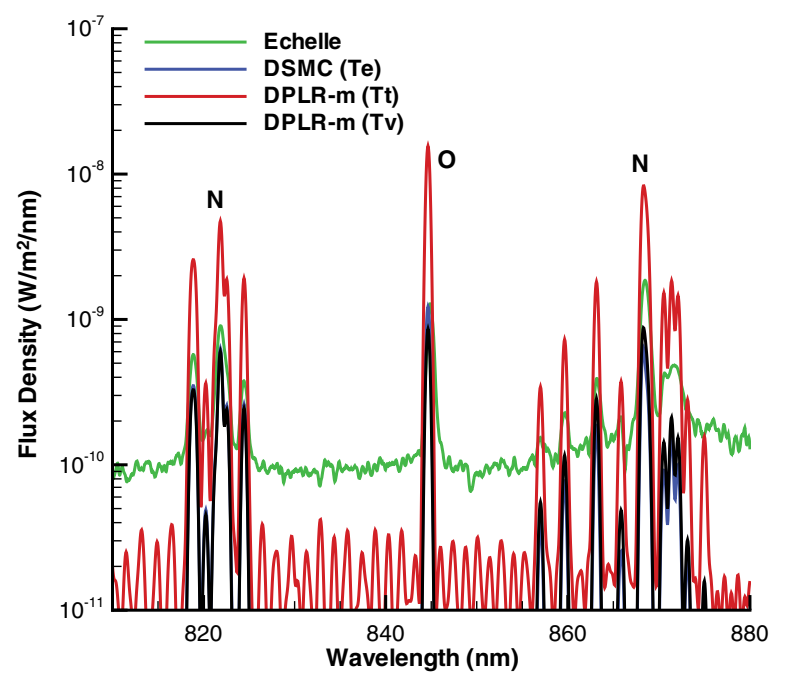

Fig. 14 Comparisons of measured and computed spectra focusing on air atomic lines at $81 \mathrm{~km}$ : DPL $\mathrm{R}-\mathrm{m}=$ modified chemistry. 


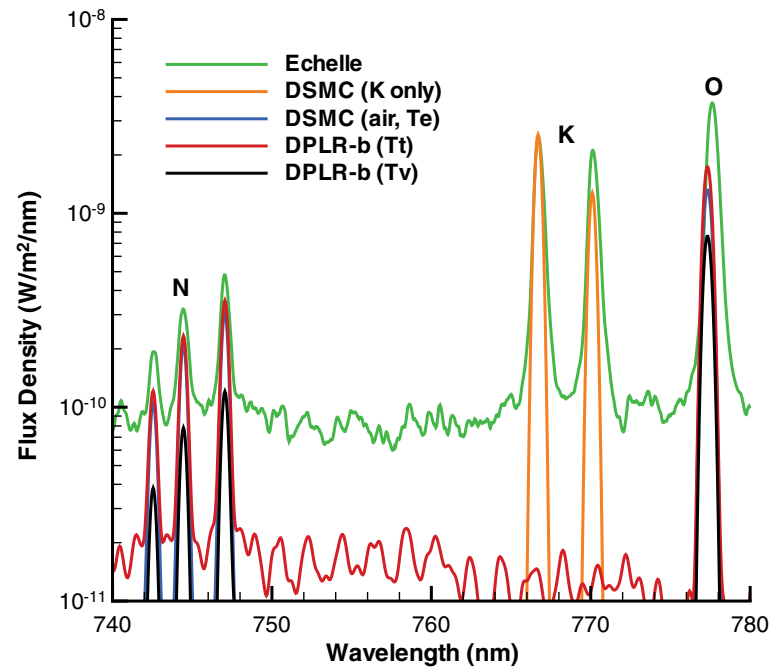

Fig. 15 Comparisons of measured and computed spectra focusing on air and potassium atomic lines at $81 \mathrm{~km}$ : DPL R-b = baseline chemistry.

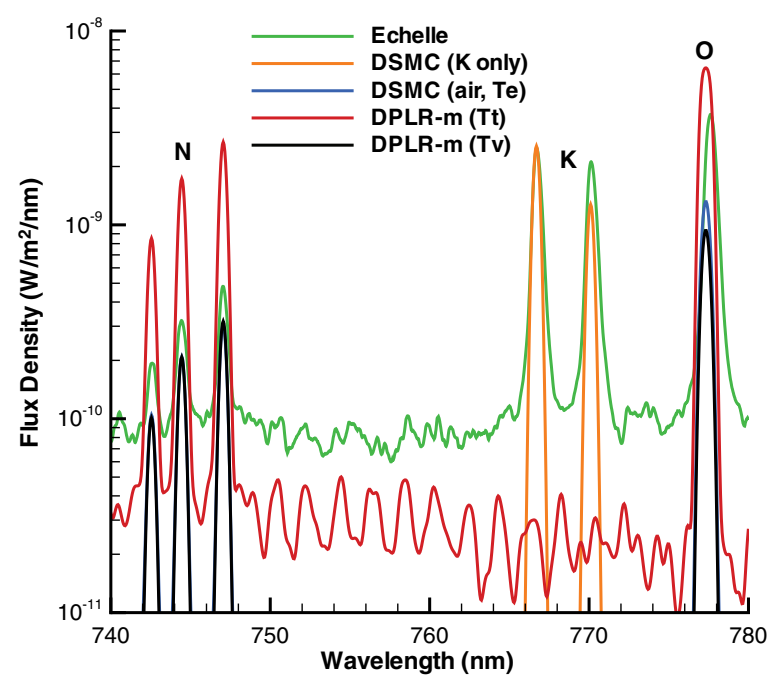

Fig. 16 Comparisons of measured and computed spectra focusing on air and potassium atomic lines at $81 \mathrm{~km}$ : DPL $\mathrm{R}-\mathrm{m}=$ modified chemistry.

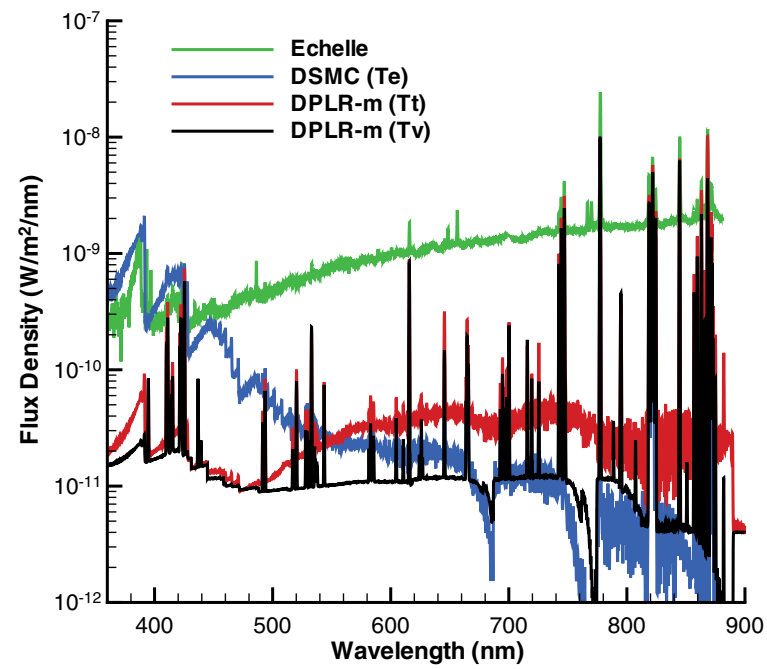

Fig. 17 Comparisons of measured and computed spectra across the full range of the Echelle instrument at $71 \mathrm{~km}$ : DPL R-m = modified chemistry.

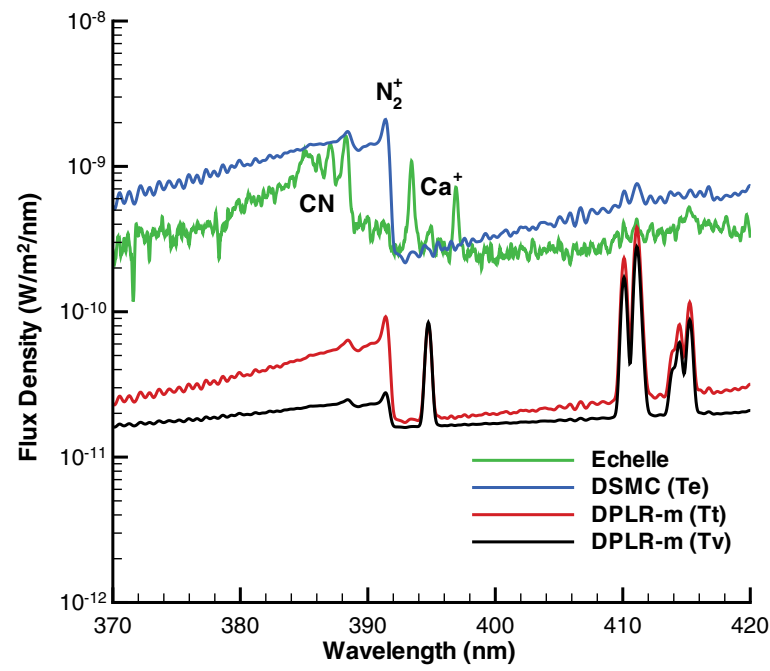

Fig. 18 Comparisons of measured and computed spectra at $71 \mathrm{~km}$ focusing on air molecular bands: DPL R-m = modified chemistry.

In Fig. 18, spectra are shown in the range of 370-420 nm. In comparison to the Echelle data, the DSMC spectrum again overpredicts the $\mathrm{N}_{2}^{+}$bandhead, whereas the DPLR spectra underpredict the feature.

In Fig. 19, spectra are shown in the range of $810-880 \mathrm{~nm}$ that contains several lines of atomic nitrogen and oxygen. The comparisons between predicted and measured spectra are not always consistent for the various features. In most cases, the DPLR result obtained with $T_{e}=T_{t}$ is in good agreement with the measured spectra and the result obtained with $T_{e}=T_{v}$ is a little lower. In general, the DSMC-based results lie below the measured emission. Detailed analysis of the computed spectra indicate that the emissions depend on the number densities of atoms and electrons, as well as the electron temperature. The primary reason for the lower atomic emissions predicted by DSMC originate in the significantly lower electron number density in the postshock region, as illustrated in Fig. 4. The reduction in emission due to lower electron number density is partially offset by higher electron temperatures predicted by DSMC. By comparison, the DPLR and DSMC levels of atomic number densities are in relatively good agreement [8]. Overall, at $71 \mathrm{~km}$, the DSMC atomic emissions tend to be about a factor of 2 lower than the corresponding DPLR results. Similar to $\mathrm{N}_{2}^{+}$, the significant differences between the DPLR and DSMC predictions of the electron number density indicates a requirement for detailed analysis of the electron chemistry modeling.

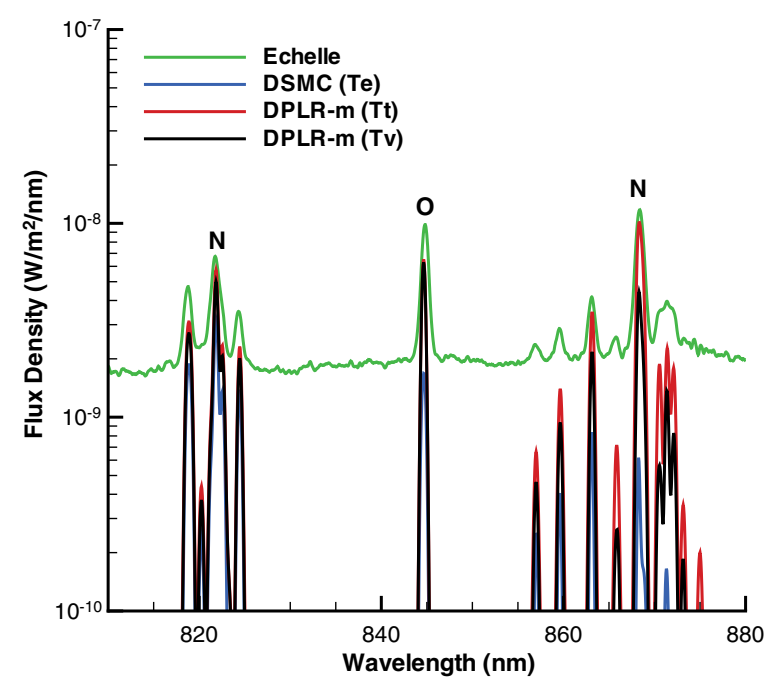

Fig. 19 Comparisons of measured and computed spectra at $71 \mathrm{~km}$ focusing on air atomic lines: DPL R-m = modified chemistry. 


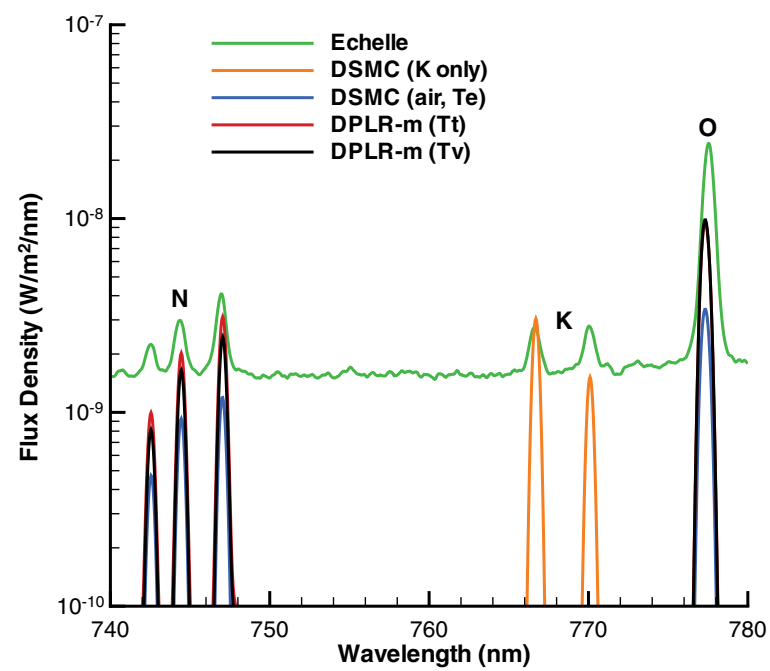

Fig. 20 Comparisons of measured and computed spectra at $71 \mathrm{~km}$ focusing on air and potassium atomic lines: DPL R-m $=$ modified chemistry.

Similar comparisons are made in the wavelength range of 740$780 \mathrm{~nm}$ in Fig. 20. Again, focusing here on the air atomic lines, the conclusions are similar to those stated earlier for Fig. 19, although all prediction methods underpredict the peak intensities of most of the atomic air lines.

\section{B. Emissions from Metal Species}

The Echelle spectra at nearly all altitudes show strong signals from several metallic atoms, including potassium, zinc, and sodium. It is believed that some of these metals originate in the white paint coating the SRC (used for thermal control of the vehicle during space flight) and some come from the PICA. To study the generation of these emissions, a series of DSMC computations is performed in which a flux of metal atoms is assumed to out-gas at the wall temperature from all surfaces of the SRC forebody at a radially uniform rate. Lineof-sight profiles of the resulting flowfield are extracted and input into NEQAIR which is run using the Boltzmann option because the rates required for QSS analysis for the excited states of these metals are not available in the literature. The Boltzmann option assumed for computation of emissions from the metal atoms is known to overpredict air emissions. However, the peak emission from the metal impurities occurs immediately adjacent to the surface due to the steep density gradient of these species. Because this is the location where the flow is closest to being in equilibrium, due to the relatively high total number density there, it is likely that the Boltzmann approach for the metal species is a better approximation than it is for the air species. Through comparison of the computed spectra with the Echelle data, the out-gas flux of each metal is adjusted until good agreement is obtained between measurement and simulation. Radial variation in these spectra is again observed and the results presented are area averaged across the SRC forebody.

Again, the spectra at $81 \mathrm{~km}$ are considered first. The Echelle data shown in Figs. 15 and 16 indicate a doublet originating in transitions from the ${ }^{2} P_{3 / 2,1 / 2}$ excited states to the ${ }^{2} S_{1 / 2}$ ground state of potassium at wavelengths of 766.70 and $770.10 \mathrm{~nm}$, respectively. The DSMC results provide very good agreement with the measured data. The degeneracy of the lower wavelength state is twice that of the higher wavelength state, and this difference scales almost directly into the Boltzmann model results for the emissions from the two states. The measured spectrum shows a reduction in intensity of the higher wavelength transition that is close to the theoretical factor of 2 . The uniform surface flux required in the DSMC computation to obtain the agreement shown in these figures is $1.35 \times 10^{20}$ particles $/ \mathrm{m}^{2} / \mathrm{s}$. Numerical studies of PICA ablation performed at NASA Ames Research Center indicate a total mass flux of material blowing off the surface at this flow condition of $1.26 \times 10^{-2} \mathrm{~kg} \cdot \mathrm{m}^{-2} \cdot \mathrm{s}^{-1}$. Thus, the required potassium flux corresponds to a blowing mass fraction of about $689 \times 10^{-6}$. Analysis of virgin PICA using a mass spectrometer, also conducted at NASA Ames Research Center, indicates that the mass fraction of potassium in PICA is in the range of $25-50 \times 10^{-6}$. Hence, the enhanced rate of potassium flux from the surface is likely not from PICA alone. It is reasonable to associate the additional flux with the evaporation of the white paint (Z-93P) applied on top of the PICA for thermal control. It should be noted that this paint contains potassium silicate binder.

A similar study is conducted for zinc and the results are shown in Fig. 21. The transition in this case is a singlet from the ${ }^{1} D_{2}$ to the ${ }^{1} P_{1}$ excited states occurring at $636.23 \mathrm{~nm}$. The measured line intensity is reproduced well using a DSMC flowfield generated with a uniform surface flux of $9.29 \times 10^{20}$ particles $/ \mathrm{m}^{2} / \mathrm{s}$, which is much higher than the potassium out-gassing rate. This flux of zinc corresponds to a blowing mass fraction of about $8270 \times 10^{-6}$. The mass spectrometer analysis of PICA indicates that the mass fraction of zinc is about $3 \times 10^{-6}$. Clearly, the high level of zinc emission observed in the Echelle spectra does not originate in the PICA material, and presumably again comes from the paint that includes zinc oxide pigment. It is noted in passing that the Echelle spectrum includes strong emission from a triplet of zinc lines centered around $472 \mathrm{~nm}$. Unfortunately, NEQAIR cannot predict intensities for these lines because no transition probabilities for them could be found in the literature.

The third metal considered is sodium, for which the final results are shown in Fig. 22. Similar to potassium, the spectral feature involves a doublet originating in transitions at 589.16 and $589.76 \mathrm{~nm}$ from the ${ }^{2} P_{3 / 2,1 / 2}$ excited states to the ground state, with the same factor of 2 difference in degeneracy that is almost perfectly reproduced in the measured signal. The required surface flux for sodium is $1.86 \times 10^{19}$ particles $/ \mathrm{m}^{2} / \mathrm{s}$, which corresponds to a blowing mass fraction of $52 \times 10^{-6}$. The mass spectrometer analysis of PICA reports sodium mass fractions ranging from about 40 to $300 \times 10^{-6}$. In this case, the required surface flux of sodium lies close enough to the measured range that it is reasonable to conclude that the PICA TPS is the most likely source of this metal impurity.

The Echelle spectrum at $71 \mathrm{~km}$ again shows two clear potassium lines in Fig. 20. The predicted total mass flux of ablated PICA material at this altitude is $9.86 \times 10^{-3} \mathrm{~kg} \cdot \mathrm{m}^{-2} \cdot \mathrm{s}^{-1}$. Using the same blowing mass fraction of potassium that was employed to obtain good agreement with the Echelle potassium data at $81 \mathrm{~km}$, the DSMC result in Fig. 20 again matches the peak emission measured at the lower wavelength. The predicted peak for the higher wavelength feature is underpredicted in comparison to the measured data.

Using the same zinc blowing mass fraction employed at $81 \mathrm{~km}$, the results produced at $71 \mathrm{~km}$ are shown in Fig. 23. The Echelle data do not show any signal from zinc at $71 \mathrm{~km}$ and this is qualitatively

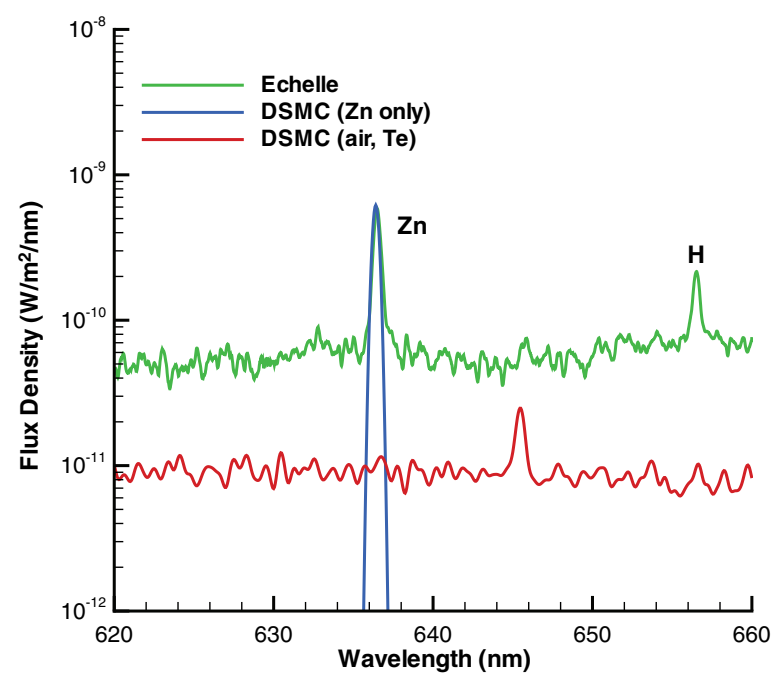

Fig. 21 Comparisons of measured and computed spectra focusing on a zinc atomic line at $81 \mathrm{~km}$. 


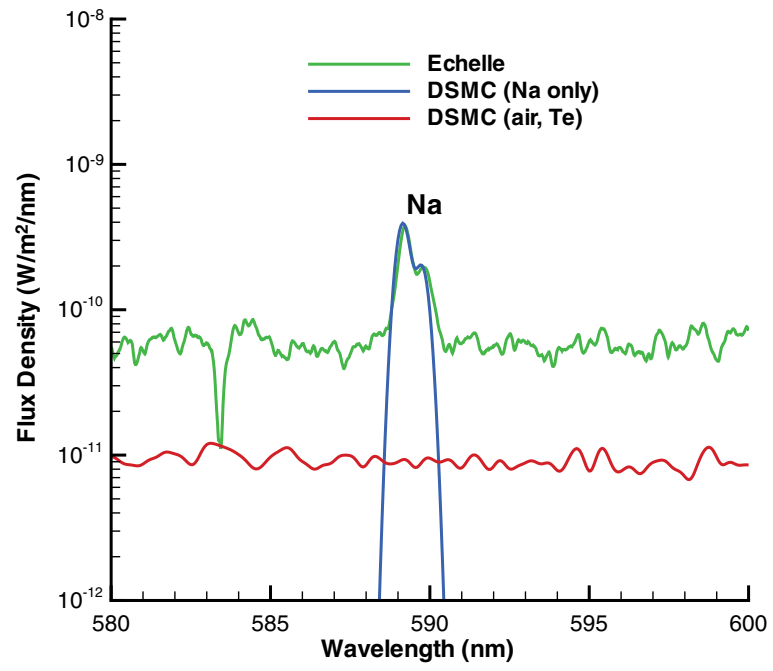

Fig. 22 Comparisons of measured and computed spectra focusing on sodium atomic lines at $81 \mathrm{~km}$.

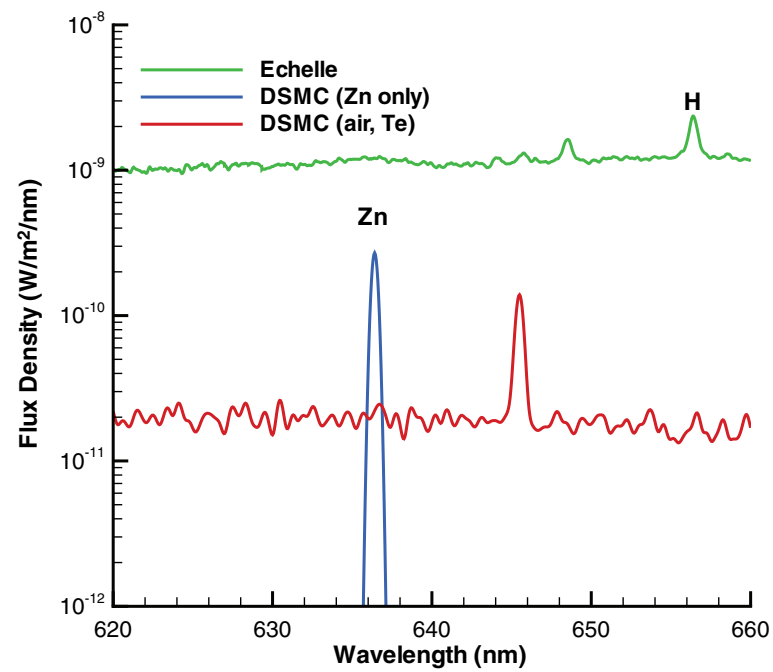

Fig. 23 Comparisons of measured and computed spectra at $71 \mathrm{~km}$ focusing on a zinc atomic line.

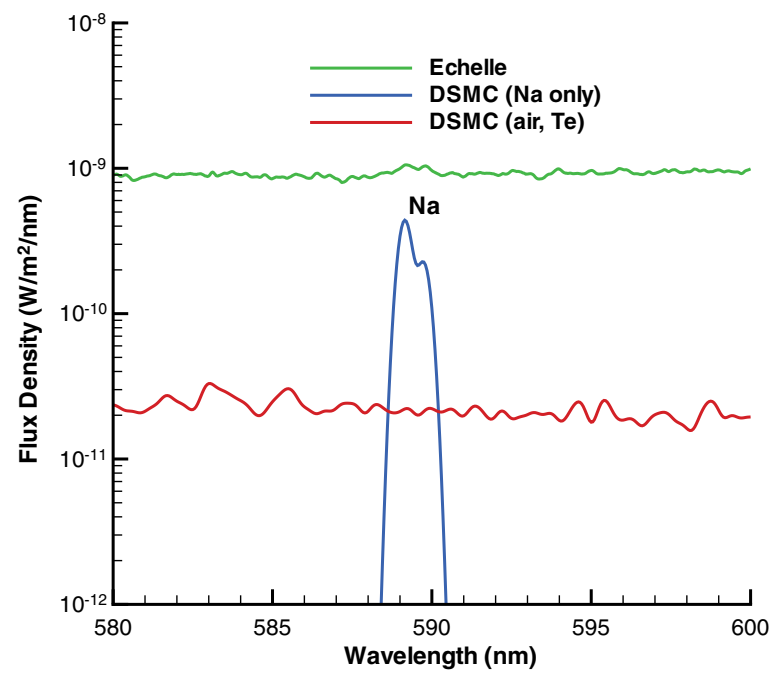

Fig. 24 Comparisons of measured and computed spectra at $71 \mathrm{~km}$ focusing on sodium atomic lines. consistent with the DSMC result. Finally, the spectra for sodium are shown in Fig. 24. Similar to zinc, employing the same mass fraction here as was used at $81 \mathrm{~km}$ leads to a predicted signal from sodium that lies below the background signal in the Echelle data. Once again, all that can be said is that the DSMC prediction is qualitatively consistent with the lack of the sodium feature in the measured spectrum.

\section{Conclusions}

The high-altitude portion of the Stardust entry trajectory generated very high-energy, strongly nonequilibrium flow conditions. In the present study, both continuum (DPLR) and particle (DSMC) simulation methods were employed to generate flowfield solutions for Stardust at altitudes of 81 and $71 \mathrm{~km}$. At high altitude, the DPLR results were sensitive to chemistry modeling. In addition, noncontinuum effects at the vehicle surface led to differences between the DSMC and modified DPLR results. At lower altitude, the DPLR results became insensitive to chemistry modeling. However, significant differences between DSMC and DPLR were still found at $71 \mathrm{~km}$ for the number densities of $\mathrm{N}_{2}^{+}$and electrons.

The DPLR and DSMC flowfield results were used as input to the nonequilibrium radiation code NEQAIR to compute emission spectra. NEQAIR requires as input all the species number densities and the translational, rotational, vibrational, and electron temperatures. The DPLR computations do not explicitly simulate an electron temperature, and so spectra were computed by assuming that the electron temperature is given by either the translational temperature or the vibrational temperature. As expected, the emissions computed using the translational temperature were significantly higher than those obtained using the vibrational temperature.

At both flight conditions, lines of sight extracted along different radial coordinates in the flowfield and input into NEQAIR produced varying levels of emission. Although the spectra computed using DPLR and the translational temperature displayed modest variations with radial location, the DPLR spectra based on vibrational temperature and the DSMC spectra, which are based on electron temperature, showed variations by factors of $2-4$. These variations are mainly explained by the fact that both the vibrational temperature and electron temperature tend to increase with radial distance from the axis. In addition, the axial extent of high levels of vibrational and electron temperature increase with radius. Each of these effects is created by the nonequilibrium phenomena present in these relatively low-density flow conditions. The radially varying spectra were weighted by area to make quantitative comparisons with the spectra measured during the Stardust entry by the Echelle instrument.

At $81 \mathrm{~km}$, for atomic air lines, three different flowfield simulations gave good agreement with the measured features: 1) DSMC, 2) baseline DPLR setting the electron temperature for NEQAIR to the overall translational temperature, and 3) modified DPLR setting the electron temperature for NEQAIR to the overall vibrational temperature. The fact that similar spectra were obtained from three different flowfields that show significant differences is both encouraging and disturbing. Analysis of the spectra reveals that the significantly higher electron number densities predicted by the baseline DPLR simulation are counterbalanced in terms of emission by the lower temperatures predicted in this same computation.

The spectral analyses of air species conducted at $71 \mathrm{~km}$ showed significantly less sensitivity to chemistry modeling in the DPLR results. In general, the DPLR-based spectra provided better comparison to the air-species features present in the measured spectra than did the DSMC-based spectra.

Analysis of the $\mathrm{N}_{2}^{+}$system revealed good agreement between the measured data and radiation predictions based on both DSMC and DPLR with the modified chemistry model. For atomic features at $71 \mathrm{~km}, \mathrm{DSMC}$ tended to significantly underpredict the emission in comparison to the measured data, and this trend was traced to the lower levels of electron number density computed by DSMC in comparison to DPLR. Thus, it is concluded that the present comparisons between computed and measured data are able to provide guidance on aspects of the modeling of the high-energy 
chemical kinetics in both DSMC and DPLR that require further study.

An interesting aspect of the Echelle measurements involves very strong emission signals from several different metallic lines. Additional DSMC computations were performed at $81 \mathrm{~km}$ in which fluxes from the Stardust surface of potassium, zinc, and sodium were included. By extracting lines of sight from the resulting flowfields, and employing the Boltzmann emission feature of NEQAIR, spectra were obtained for these metals. The magnitudes of the metal fluxes from the surface were varied until good agreement was found between the computed and measured line intensities. These studies indicated that the surface fluxes for potassium and zinc, which were required to generate the emissions measured by Echelle, are much higher than can be accounted for by impurities in the PICA thermal protection material employed on the Stardust capsule. It is therefore concluded that these emissions originate in the white paint that covered the PICA, which is known to contain both potassium silicate and zinc oxide. For sodium, the flux inferred from the simulations was on the same order of magnitude as the impurity concentration for virgin PICA, and so this signal is concluded to arise from ablation of the TPS material.

At $71 \mathrm{~km}$, use of the same mass fractions for blowing from the surface that were established in the $81 \mathrm{~km}$ analysis led to good agreement with the measured potassium signal. For zinc and sodium, no clear features were measured at $71 \mathrm{~km}$. This finding is in qualitative agreement with the simulation results that predicted the signals from these metals to lie below the measured background intensity.

\section{Acknowledgments}

The work of the first author was sponsored in part by the Stanford Center for Turbulence Research and the Eloret Corporation through NASA contract NNA04BC25C. The first author also gratefully acknowledges the assistance of Kerry Trumble in obtaining the DPLR solutions, Dinesh Prabhu in obtaining the NEQAIR results, Ioanna Cozmuta for predictions of PICA blowing rates, and Mairead Stackpoole for measurements of the chemical composition of PICA.

\section{References}

[1] Olynick, D., Chen, Y.-K., and Tauber, M. E., "Aerothermodynamics of the Stardust Sample Return Capsule," Journal of Spacecraft and Rockets, Vol. 36, No. 3, 1999, pp. 442-462. doi: $10.2514 / 2.3466$

[2] Mitcheltree, R. A., Wilmoth, R. G., Cheatwood, F. M., Brauckmann, G. J., and Greene, F. A., "Aerodynamics of Stardust Sample Return Capsule," Journal of Spacecraft and Rockets, Vol. 36, No. 3, 1999 , pp. 429-435. doi: $10.2514 / 2.3463$

[3] Wilmoth, R. G., Mitcheltree, R. A., and Moss, J. N., "Low-Density Aerodynamics of the Stardust Sample Return Capsule," Journal of Spacecraft and Rockets, Vol. 36, No. 3, 1999, pp. 436-441. doi: $10.2514 / 2.3464$

[4] Gupta, R. N., "Aerothermodynamic Analysis of Stardust Sample Return Capsule with Coupled Radiation and Ablation," Journal of Spacecraft and Rockets, Vol. 37, No. 4, 2000, pp. 507-514. doi:10.2514/2.3592

[5] Bird, G. A., Molecular Gas Dynamics and the Direct Simulation of Gas Flows, Oxford Univ. Press, Oxford, England, U.K., 1994.

[6] Ozawa, T., Zhong, J., Levin, D. A., Boger, D., and Wright, M. J., "Modeling of the Stardust Reentry Flows with Ionization in DSMC,"
AIAA Paper 2007-0611, Jan. 2007.

[7] Boyd, I. D., Trumble, K. A., and Wright, M. J., "Nonequilibrium Particle and Continuum Analyses of Stardust Entry for NearContinuum Conditions," AIAA Paper 2007-4543, June 2007.

[8] Boyd, I. D., Trumble, K. A., and Wright, M. J., "Modeling of Stardust Entry at High Altitude, Part 1: Flow Field Analysis," 2008, (submitted to Journal of Spacecraft and Rockets).

[9] Whiting, E. E., Park, C., Liu, Y., Arnold, J. O., and Paterson, J. A., "NEQAIR96, Nonequilibrium and Equilibrium Radiative Transport and Spectra Program: User's Manual," NASA Reference Publ. 1389, Dec. 1996.

[10] Jenniskens, P., "Observations of the STARDUST Sample Return Capsule Entry with a Slit-Less Echelle Spectrograph," AIAA Paper 2008-1210, Jan. 2008.

[11] Wright, M., Loomis, M., and Papadopoulos, P., "Aerothermal Analysis of the Project Fire II Afterbody Flow," Journal of Thermophysics and Heat Transfer, Vol. 17, No. 2, 2003, pp. 240-249. doi: $10.2514 / 2.6757$

[12] Wright, M., Candler, G., and Bose, D., "Data-Parallel Line Relaxation Method for the Navier-Stokes Equations," AIAA Journal, Vol. 36, No. 9, 1998, pp. 1603-1609. doi: $10.2514 / 2.586$

[13] Parker, J. G., "Rotational and Vibrational Relaxation in Diatomic Gases," Physics of Fluids, Vol. 2, No. 4, 1959, pp. 449-462. doi:10.1063/1.1724417

[14] Millikan, R., and White, D., "Systematics of Vibrational Relaxation," Journal of Chemical Physics, Vol. 39, No. 12, 1963, pp. 3209-3213. doi:10.1063/1.1734182

[15] Park, C., Nonequilibrium Hypersonic Aerothermodynamics, Wiley, New York, 1990.

[16] Park, C., "Review of Chemical-Kinetic Problems of Future NASA Missions, I: Earth Entries," Journal of Thermophysics and Heat Transfer, Vol. 7, No. 3, 1993, pp. 385-398. doi: $10.2514 / 3.431$

[17] Bose, D., and Candler, G., "Thermal Rate Constants of the $\mathrm{N} 2+\mathrm{O}=$ ED NO + N Reaction Using Ab Initio ${ }^{3} A_{\prime \prime}$ and ${ }^{3} A$, Potential Energy Surfaces," Journal of Chemical Physics, Vol. 104, No. 8, 1996, pp. $2825-2833$. doi:10.1063/1.471106

[18] Boyd, I. D., and Gokcen, T., "Computation of Axisymmetric and Ionized Hypersonic Flows Using Particle and Continuum Method," AIAA Journal, Vol. 32, No. 9, 1994, pp. 1828-1837. doi: $10.2514 / 3.12180$

[19] Boyd, I. D., "Analysis of Rotational Nonequilibrium in Standing Shock Waves of Nitrogen," AIAA Journal Vol. 28, No. 11, 1990, pp. $1997-$ 1999. doi: $10.2514 / 3.10511$

[20] Boyd, I. D., "Analysis of Vibrational-Translational Energy Transfer Using the Direct Simulation Monte Carlo Method," Physics of Fluids A, Vol. 3, No. 7, 1991, pp. 1785-1791. doi:10.1063/1.857959

[21] Haas, B. L., and Boyd, I. D., "Models for Direct Monte Carlo Simulation of Coupled Vibration-Dissociation," Physics of Fluids A, Vol. 5, No. 2, 1993, pp. 478-489. doi: $10.1063 / 1.858870$

[22] Wilson, J., "Ionization Rate of Air Behind High-Speed Shock Waves," Physics of Fluids, Vol. 9, No. 10, 1966, pp. 1913-1921. doi:10.1063/1.1761543

[23] Boyd, I. D., "Modeling Backward Chemical Rate Processes in the Direct Simulation Monte Carlo Method," Physics of Fluids, Vol. 19 No. 12, 2007, pp. 126103.1-126103.9. doi:10.1063/1.2825038 